\title{
Why We Need to Use 3D Fourier Transform Analysis to Evaluate a High- performance TEM
}

\author{
Kazuo Ishizuka ${ }^{1,3}$ and Koji Kimoto ${ }^{1,2}$ \\ 1. National Institute for Materials Science, 1-1 Namiki, Tsukuba, Ibaraki, Japan \\ 2. Department of Applied Chemistry, Kyushu University, 1-1 Namiki, Tsukuba, Ibaraki, Japan \\ 3. HREM Research Inc., 14-48 Matsukazedai, Higashimatsuyama, Saitama, Japan
}

Correcting the spherical aberration (Cs) of the objective lens was realized just before the millennium for HRTEM [1] and for dedicated scanning TEM [2]. Furthermore, a corrector for the chromatic aberration (Cc) was realized using ultra-stable electronics [3]. When the resolution becomes sub-angstrom by correcting the Cs, the information limit is determined mainly by partial temporal coherence. Thus, a method to measure the partial temporal coherence becomes important more than ever. Since a traditional Young's fringe test does not reveal the information limit, a new method to evaluate the focus spread, and thus temporal coherence was proposed based on a low-pass filtered tilted-beam diffractogram [4]. More recently, information transfer in a Cs/Cc corrected TEM was discussed, and the focus spread for a $\mathrm{Cs} / \mathrm{Cc}$ corrected microscope was measured from a tilted-beam diffractogram envelope [5].

However, in order to observe an actual information transfer during the image formation down to a few ten pm, we need a strong scattering amorphous object made from heavy elements or a thick amorphous sample, both of which will introduce pronounced non-linear contribution. Therefore, we proposed the 3D Fourier transform (FT) of through-focus TEM images to extract linear contribution from a non-linear image [6]. With this method we evaluated the performances of Cs-corrected TEM at lower-voltage $(80 \mathrm{kV})$. Here, we used through-focus images taken with axial illumination, and thus the estimated value includes also the isotropic damping factors. Then, we extended the 3D FT method to quantitatively measure temporal coherence using tilted-beam through-focus images, where we could compensate attenuation from the isotropic damping factors [7]. We evaluated the performances of some Cs-corrected low-voltage TEMs equipped with monochromator.

In this report we compare the 3D FT analysis with the tilted-beam diffractogram analysis (2D FT analysis), and clarify the necessity to use the 3D FT analysis to evaluate a high-performance TEM. We may note firstly that the diffractogram analysis cannot extract linear image information out from the image intensity. Furthermore, we have to make use of a weak scattering approximation, since the diffractogram cannot separate two linear image contributions. Moreover, the tilted-beam diffractogram becomes broad for the case of a small defocus spread as shown in Figure 1 (b). Thus, the diffractogram analysis becomes difficult for a Cc-corrected microscope or a microscope with a monocromator. Using the 3D FT analysis we can use a strong scattering object, since we can analyze each linear term separately as explained below.

We have derived the 3D transmission cross coefficient (TCC) for the 3D FT of the through-focus TEM images. The 3D TCC is a Fourier transform along the z-direction of the (2D) TCC as derived in [8]:

$$
T_{2}\left(\mathbf{g}_{2}+\boldsymbol{\tau}_{\mathbf{2}}, \boldsymbol{\tau}_{2} ; z\right) \approx \exp \left\{-2 \pi i\left[\chi\left(\mathbf{g}_{2}+\boldsymbol{\tau}_{2} ; z\right)-\chi\left(\boldsymbol{\tau}_{2} ; z\right)\right]\right\} E_{t}\left(\mathbf{g}_{2}+\boldsymbol{\tau}_{2}, \boldsymbol{\tau}_{\mathbf{2}}\right) \cdot E_{s}\left(\mathbf{g}_{2}+\boldsymbol{\tau}_{\mathbf{2}}, \boldsymbol{\tau}_{\mathbf{2}} ; z\right) .
$$

where $E_{t}\left(\mathbf{g}_{2}, \mathbf{g}_{2}^{\prime}\right)=\exp \left\{-(\pi \Delta \lambda / 2)^{2}\left(\mathrm{~g}_{2}^{2}-\mathrm{g}_{2}^{\prime 2}\right)^{2}\right\}$ and $E_{s}\left(\mathbf{g}_{2}, \mathbf{g}_{2}^{\prime} ; z\right)=\exp \left\{-\left(\pi q_{0}\right)^{2}\left(\nabla \chi\left(\mathbf{g}_{2} ; z\right)-\nabla \chi\left(\mathbf{g}^{\prime} ; z\right)\right)^{2}\right\}$

represent the temporal and spatial envelopes, respectively. Then, the 3D TCC will be written by

$$
\begin{aligned}
& T_{3}\left(\mathbf{g}_{2}+\boldsymbol{\tau}_{2}, \boldsymbol{\tau}_{2}, w\right) \approx \exp \left\{-2 \pi i\left[\hat{\boldsymbol{\chi}}\left(\mathbf{g}_{2}+\boldsymbol{\tau}_{2}\right)-\hat{\chi}\left(\boldsymbol{\tau}_{2}\right)\right]\right\} E_{t}\left(\mathbf{g}_{2}+\boldsymbol{\tau}_{2}, \boldsymbol{\tau}_{2}\right) \\
& \quad \times \exp \left\{-2 \pi i\left[\left|\nabla \hat{\chi}_{/ /}\left(\mathbf{g}_{2}+\boldsymbol{\tau}_{\mathbf{2}}\right)-\nabla \hat{\chi}_{/ /}\left(+\boldsymbol{\tau}_{2}\right)\right| / \lambda g_{2}\right] \hat{w}\right\} \\
& \quad \times \exp \left\{-\left(\boldsymbol{\pi} q_{0}\right)^{2}\left(\nabla \hat{\chi}_{\perp}\left(\mathbf{g}_{2}+\boldsymbol{\tau}_{\mathbf{2}}\right)-\nabla \hat{\chi}_{\perp}\left(\boldsymbol{\tau}_{2}\right)\right)^{2}\right\} E_{\text {Ewald }}\left(\mathbf{g}_{2}+\boldsymbol{\tau}_{\mathbf{2}}, \boldsymbol{\tau}_{\mathbf{2}}, w\right)
\end{aligned}
$$


where $\chi\left(\mathbf{g}_{2} ; z\right)=\hat{\chi}\left(\mathbf{g}_{2}\right)+\frac{1}{2} \lambda z \mathbf{g}_{2}^{2}$, and $E_{\text {Ewald }}\left(\mathbf{g}_{2}+\boldsymbol{\tau}_{\mathbf{2}}, \boldsymbol{\tau}_{\mathbf{2}}, w\right)=\frac{1}{\sqrt{\pi} q_{0} \lambda g_{2}} \exp \left[-\frac{1}{\left(q_{0} \lambda g_{2}\right)^{2}} \hat{w}^{2}\left(\mathbf{g}_{2}+\boldsymbol{\tau}_{\mathbf{2}}, \boldsymbol{\tau}_{\mathbf{2}}, w\right)\right]$ represents the Ewald sphere, which is a normalized Gaussian. Here, $\hat{w}$ represents a distance measured from the Ewald sphere along the w-axis. Figure 2 shows an example of the Ewald spheres. Using the Ewald spheres we can extract linear image information from the image intensity, and further separate two linear image contributions Thus, we can evaluate the temporal envelope on the sharp Ewald spheres, even when the temporal envelopes become broad for the case of a small defocus spread as shown in Fig. 1. Therefore, we can use a thick sample or a sample made from strong scattering elements, even when the dynamical/multiple scattering becomes significant. This is the necessary condition if we want to directly observe the linear image transfer up to high angles. Furthermore, our method using 3D FT of the through-focus images gives a possibility to directly observe the distribution of the focus spread via a Fourier transform of the measured temporal envelope for a high-performance microscope [9].

\section{References:}

[1] M. Haider, et al, Nature 392 (1998) p.768.

[2] K. Krivanek, N. Dellby, A. Lupini, Ultramicroscopy 78 (1999), p.1.

[3] M. Haider, et al, Microsc Microanal 13(S2) (2007), p.1156CD.

[4] J. Barthel, A. Thust, Physical Review Letters 101 (2008), p.200801.

[5] M. Haider, et al, Micros. Microanal. 16 (2010), p.393.

[6] K Kimoto, et al, Ultramicroscopy 121 (2012), p.31.

[7] K Kimoto, et al, Ultramicroscopy 134 (2013), p.86.

[8] K. Ishizuka, Ultramicroscopy 5 (1980), p.55.

[9] This study was partly supported by the JST Research Acceleration Program and the Nano Platform Program of MEXT, Japan.

(a)
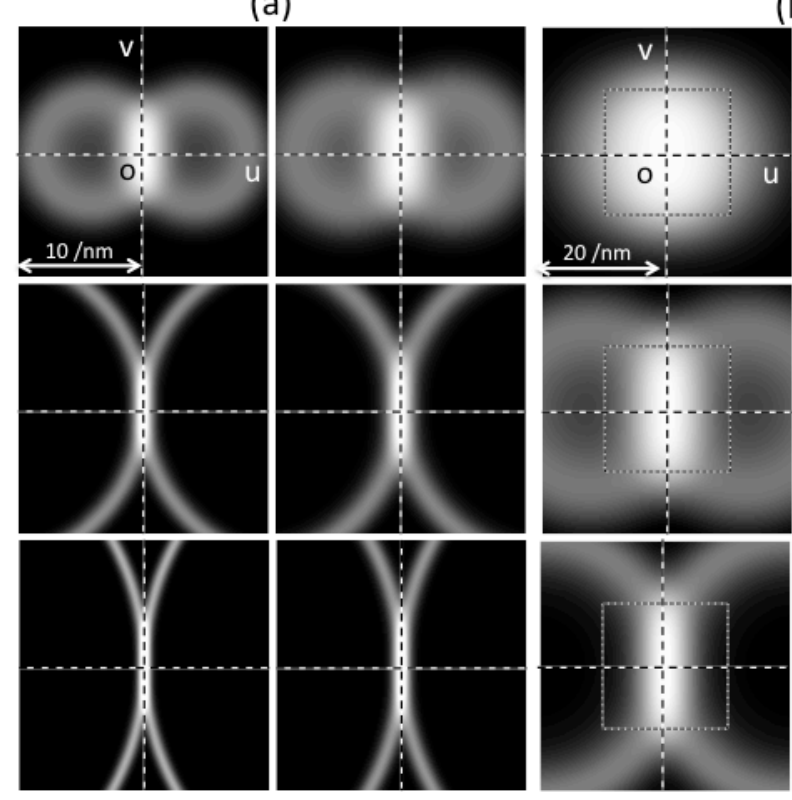

(b)

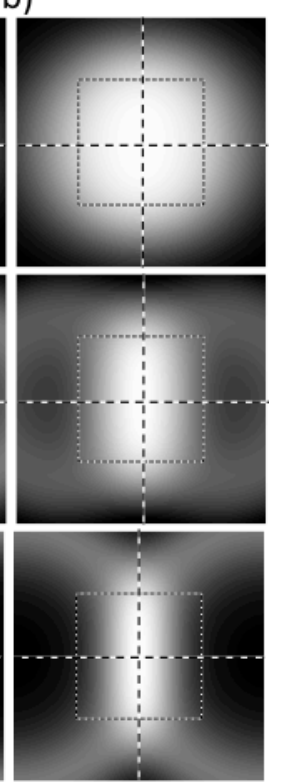

Figure 1. Temporal damping of tilted-beam diffractograms for defocus spreads of (a) $5 \mathrm{~nm}$ and (b) $0.5 \mathrm{~nm}$ with $80 \mathrm{kV}$ electrons. Left and right columns show low-pass filtered diffractograms and diffractogram envelopes. The tilt angles from the top are 1,3 and 5 degrees.
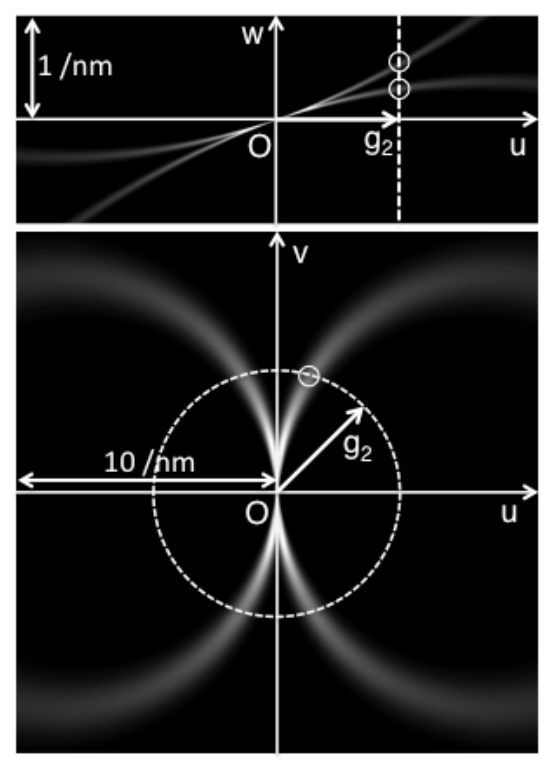

Figure 2. uv and uw sections of the Ewald spheres for a 2-mrad beam convergence with $80 \mathrm{kV}$ electrons. 